EUROPEAN LABORATORY FOR PARTICLE PHYSICS

CERN-PPE/97-003

10th January 1997

\title{
The Topology Dependence of Charged Particle Multiplicities in Three-Jet Events
}

The ALEPH Collaboration

\begin{abstract}
A study of individual jet and whole-event charged particle multiplicities in three-jet events measured in $e^{+} e^{-}$annihilation at the $Z$ reveals a significant topology dependence. Mean jet multiplicities are inadequately described by jet energies; interjet angles must also be specified. Quantitative tests suggest that it is necessary to use transverse-momentum-like scales to describe the data.
\end{abstract}

To be submitted to Zeitschrift für Physik $C$ 


\section{The ALEPH Collaboration}

R. Barate, D. Buskulic, D. Decamp, P. Ghez, C. Goy, J.-P. Lees, A. Lucotte, M.-N. Minard, J.-Y. Nief, P. Odier, B. Pietrzyk

Laboratoire de Physique des Particules (LAPP), IN $N^{2} P^{3}$-CNRS, 74019 Annecy-le-Vieux Cedex, France

M.P. Casado, M. Chmeissani, P. Comas, J.M. Crespo, M. Delfino, E. Fernandez, M. Fernandez-Bosman, Ll. Garrido, ${ }^{15}$ A. Juste, M. Martinez, R. Miquel, Ll.M. Mir, S. Orteu, C. Padilla, I.C. Park, A. Pascual, J.A. Perlas, I. Riu, F. Sanchez, F. Teubert

Institut de Fisica d'Altes Energies, Universitat Autonoma de Barcelona, 08193 Bellaterra (Barcelona), Spain $^{7}$

A. Colaleo, D. Creanza, M. de Palma, G. Gelao, G. Iaselli, G. Maggi, M. Maggi, N. Marinelli, S. Nuzzo, A. Ranieri, G. Raso, F. Ruggieri, G. Selvaggi, L. Silvestris, P. Tempesta, A. Tricomi, ${ }^{3}$ G. Zito

Dipartimento di Fisica, INFN Sezione di Bari, 70126 Bari, Italy

X. Huang, J. Lin, Q. Ouyang, T. Wang, Y. Xie, R. Xu, S. Xue, J. Zhang, L. Zhang, W. Zhao

Institute of High-Energy Physics, Academia Sinica, Beijing, The People's Republic of China ${ }^{8}$

D. Abbaneo, R. Alemany, A.O. Bazarko, ${ }^{25}$ P. Bright-Thomas, M. Cattaneo, F. Cerutti, H. Drevermann, R.W. Forty, M. Frank, R. Hagelberg, J. Harvey, P. Janot, B. Jost, E. Kneringer, J. Knobloch, I. Lehraus, G. Lutters, P. Mato, A. Minten, L. Moneta, A. Pacheco, J.-F. Pusztaszeri, F. Ranjard, P. Rensing, ${ }^{12}$ G. Rizzo, L. Rolandi, D. Schlatter, M. Schmelling, ${ }^{26}$ M. Schmitt, O. Schneider, W. Tejessy, I.R. Tomalin, H. Wachsmuth, A. Wagner

European Laboratory for Particle Physics (CERN), 1211 Geneva 23, Switzerland

Z. Ajaltouni, A. Barrès, C. Boyer, A. Falvard, C. Ferdi, P. Gay, C . Guicheney, P. Henrard, J. Jousset, B. Michel, S. Monteil, J-C. Montret, D. Pallin, P. Perret, F. Podlyski, J. Proriol, P. Rosnet, J.M. Rossignol

Laboratoire de Physique Corpusculaire, Université Blaise Pascal, $I N^{2} P^{3}$-CNRS, Clermont-Ferrand, 63177 Aubière, France

T. Fearnley, J.B. Hansen, J.D. Hansen, J.R. Hansen, P.H. Hansen, B.S. Nilsson, B. Rensch, A. Wäänänen Niels Bohr Institute, 2100 Copenhagen, Denmark ${ }^{9}$

G. Daskalakis, A. Kyriakis, C. Markou, E. Simopoulou, I. Siotis, A. Vayaki, K. Zachariadou Nuclear Research Center Demokritos (NRCD), Athens, Greece

A. Blondel, G. Bonneaud, J.C. Brient, P. Bourdon, A. Rougé, M. Rumpf, A. Valassi, ${ }^{6}$ M. Verderi, H. Videau

Laboratoire de Physique Nucléaire et des Hautes Energies, Ecole Polytechnique, IN ${ }^{2} P^{3}$-CNRS, 91128 Palaiseau Cedex, France

D.J. Candlin, M.I. Parsons

Department of Physics, University of Edinburgh, Edinburgh EH9 3JZ, United Kingdom ${ }^{10}$

E. Focardi, G. Parrini

Dipartimento di Fisica, Università di Firenze, INFN Sezione di Firenze, 50125 Firenze, Italy

M. Corden, C. Georgiopoulos, D.E. Jaffe

Supercomputer Computations Research Institute, Florida State University, Tallahassee, FL 323064052, USA ${ }^{13,14}$

A. Antonelli, G. Bencivenni, G. Bologna, ${ }^{4}$ F. Bossi, P. Campana, G. Capon, D. Casper, V. Chiarella, G. Felici, P. Laurelli, G. Mannocchi, ${ }^{5}$ F. Murtas, G.P. Murtas, L. Passalacqua, M. Pepe-Altarelli

Laboratori Nazionali dell'INFN (LNF-INFN), 00044 Frascati, Italy

L. Curtis, S.J. Dorris, A.W. Halley, I.G. Knowles, J.G. Lynch, V. O'Shea, C. Raine, J.M. Scarr, K. Smith, P. Teixeira-Dias, A.S. Thompson, E. Thomson, F. Thomson, R.M. Turnbull

Department of Physics and Astronomy, University of Glasgow, Glasgow G12 8QQ,United Kingdom ${ }^{10}$ 
U. Becker, O. Buchmüller, C. Geweniger, G. Graefe, P. Hanke, G. Hansper, V. Hepp, E.E. Kluge, A. Putzer, M. Schmidt, J. Sommer, H. Stenzel, K. Tittel, S. Werner, M. Wunsch

Institut für Hochenergiephysik, Universität Heidelberg, 69120 Heidelberg, Fed. Rep. of Germany ${ }^{16}$

R. Beuselinck, D.M. Binnie, W. Cameron, P.J. Dornan, M. Girone, S. Goodsir, E.B. Martin, A. Moutoussi, J. Nash, J.K. Sedgbeer, A.M. Stacey, M.D. Williams

Department of Physics, Imperial College, London SW7 2BZ, United Kingdom ${ }^{10}$

G. Dissertori, P. Girtler, D. Kuhn, G. Rudolph

Institut für Experimentalphysik, Universität Innsbruck, 6020 Innsbruck, Austria ${ }^{18}$

A.P. Betteridge, C.K. Bowdery, P. Colrain, G. Crawford, A.J. Finch, F. Foster, G. Hughes, T. Sloan, M.I. Williams

Department of Physics, University of Lancaster, Lancaster LA1 4YB, United Kingdom ${ }^{10}$

A. Galla, I. Giehl, A.M. Greene, C. Hoffmann, K. Jakobs, K. Kleinknecht, G. Quast, B. Renk, E. Rohne, H.-G. Sander, P. van Gemmeren, C. Zeitnitz

Institut für Physik, Universität Mainz, 55099 Mainz, Fed. Rep. of Germany ${ }^{16}$

J.J. Aubert, C. Benchouk, A. Bonissent, G. Bujosa, D. Calvet, J. Carr, P. Coyle, C. Diaconu, F. Etienne, N. Konstantinidis, O. Leroy, P. Payre, D. Rousseau, M. Talby, A. Sadouki, M. Thulasidas, K. Trabelsi Centre de Physique des Particules, Faculté des Sciences de Luminy, IN ${ }^{2} P^{3}$-CNRS, 13288 Marseille, France

M. Aleppo, F. Ragusa ${ }^{2}$

Dipartimento di Fisica, Università di Milano e INFN Sezione di Milano, 20133 Milano, Italy

R. Berlich, W. Blum, V. Büscher, H. Dietl, F. Dydak, ${ }^{2}$ G. Ganis, C. Gotzhein, H. Kroha, G. Lütjens, G. Lutz, W. Männer, H.-G. Moser, R. Richter, A. Rosado-Schlosser, S. Schael, R. Settles, H. Seywerd, R. St. Denis, H. Stenzel, W. Wiedenmann, G. Wolf

Max-Planck-Institut für Physik, Werner-Heisenberg-Institut, 80805 München, Fed. Rep. of Germany ${ }^{16}$

J. Boucrot, O. Callot, ${ }^{2}$ S. Chen, Y. Choi, ${ }^{21}$ A. Cordier, M. Davier, L. Duflot, J.-F. Grivaz, Ph. Heusse, A. Höcker, A. Jacholkowska, M. Jacquet, D.W. Kim ${ }^{19}$ F. Le Diberder, J. Lefrançois, A.-M. Lutz, I. Nikolic, H.J. Park ${ }^{19}$ M.-H. Schune, S. Simion, J.-J. Veillet, I. Videau, D. Zerwas

Laboratoire de l'Accélérateur Linéaire, Université de Paris-Sud, $I N^{2} P^{3}-C N R S, 91405$ Orsay Cedex, France

P. Azzurri, G. Bagliesi, G. Batignani, S. Bettarini, C. Bozzi, G. Calderini, M. Carpinelli, M.A. Ciocci, V. Ciulli, R. Dell'Orso, R. Fantechi, I. Ferrante, L. Foà, ${ }^{1}$ F. Forti, A. Giassi, M.A. Giorgi, A. Gregorio, F. Ligabue, A. Lusiani, P.S. Marrocchesi, A. Messineo, F. Palla, G. Sanguinetti, A. Sciabà, P. Spagnolo, J. Steinberger, R. Tenchini, G. Tonelli, ${ }^{24}$ C. Vannini, A. Venturi, P.G. Verdini

Dipartimento di Fisica dell'Università, INFN Sezione di Pisa, e Scuola Normale Superiore, 56010 Pisa, Italy

G.A. Blair, L.M. Bryant, J.T. Chambers, Y. Gao, M.G. Green, T. Medcalf, P. Perrodo, J.A. Strong, J.H. von Wimmersperg-Toeller

Department of Physics, Royal Holloway \& Bedford New College, University of London, Surrey TW20 OEX, United Kingdom ${ }^{10}$

D.R. Botterill, R.W. Clifft, T.R. Edgecock, S. Haywood, P. Maley, P.R. Norton, J.C. Thompson, A.E. Wright

Particle Physics Dept., Rutherford Appleton Laboratory, Chilton, Didcot, Oxon OX11 OQX, United Kingdom ${ }^{10}$

B. Bloch-Devaux, P. Colas, S. Emery, W. Kozanecki, E. Lançon, M.C. Lemaire, E. Locci, P. Perez, J. Rander, J.-F. Renardy, A. Roussarie, J.-P. Schuller, J. Schwindling, A. Trabelsi, B. Vallage

CEA, DAPNIA/Service de Physique des Particules, CE-Saclay, 91191 Gif-sur-Yvette Cedex, France ${ }^{17}$

S.N. Black, J.H. Dann, R.P. Johnson, H.Y. Kim, A.M. Litke, M.A. McNeil, G. Taylor

Institute for Particle Physics, University of California at Santa Cruz, Santa Cruz, CA 95064, USA ${ }^{22}$ 
C.N. Booth, R. Boswell, C.A.J. Brew, S. Cartwright, F. Combley, M.S. Kelly, M. Lehto, W.M. Newton, J. Reeve, L.F. Thompson

Department of Physics, University of Sheffield, Sheffield S3 7RH, United Kingdom ${ }^{10}$

A. Böhrer, S. Brandt, G. Cowan, C. Grupen, P. Saraiva, L. Smolik, F. Stephan

Fachbereich Physik, Universität Siegen, 57068 Siegen, Fed. Rep. of Germany ${ }^{16}$

M. Apollonio, L. Bosisio, R. Della Marina, G. Giannini, B. Gobbo, G. Musolino

Dipartimento di Fisica, Università di Trieste e INFN Sezione di Trieste, 34127 Trieste, Italy

J. Rothberg, S. Wasserbaech

Experimental Elementary Particle Physics, University of Washington, WA 98195 Seattle, U.S.A.

S.R. Armstrong, P. Elmer, Z. Feng, ${ }^{20}$ D.P.S. Ferguson, Y.S. Gao ${ }^{23}$ S. González, J. Grahl, T.C. Greening, O.J. Hayes, H. Hu, P.A. McNamara III, J.M. Nachtman, W. Orejudos, Y.B. Pan, Y. Saadi, I.J. Scott, J. Walsh, Sau Lan Wu, X. Wu, J.M. Yamartino, M. Zheng, G. Zobernig

Department of Physics, University of Wisconsin, Madison, WI 53706, USA ${ }^{11}$

\footnotetext{
${ }^{1}$ Now at CERN, 1211 Geneva 23, Switzerland.

${ }^{2}$ Also at CERN, 1211 Geneva 23, Switzerland.

${ }^{3}$ Also at Dipartimento di Fisica, INFN, Sezione di Catania, Catania, Italy.

${ }^{4}$ Also Istituto di Fisica Generale, Università di Torino, Torino, Italy.

${ }^{5}$ Also Istituto di Cosmo-Geofisica del C.N.R., Torino, Italy.

${ }^{6}$ Supported by the Commission of the European Communities, contract ERBCHBICT941234.

${ }^{7}$ Supported by CICYT, Spain.

${ }^{8}$ Supported by the National Science Foundation of China.

${ }^{9}$ Supported by the Danish Natural Science Research Council.

${ }^{10}$ Supported by the UK Particle Physics and Astronomy Research Council.

${ }^{11}$ Supported by the US Department of Energy, grant DE-FG0295-ER40896.

${ }^{12}$ Now at Dragon Systems, Newton, MA 02160, U.S.A.

${ }^{13}$ Supported by the US Department of Energy, contract DE-FG05-92ER40742.

${ }^{14}$ Supported by the US Department of Energy, contract DE-FC05-85ER250000.

${ }^{15}$ Permanent address: Universitat de Barcelona, 08208 Barcelona, Spain.

${ }^{16}$ Supported by the Bundesministerium für Bildung, Wissenschaft, Forschung und Technologie, Fed. Rep. of Germany.

${ }^{17}$ Supported by the Direction des Sciences de la Matière, C.E.A.

${ }^{18}$ Supported by Fonds zur Förderung der wissenschaftlichen Forschung, Austria.

${ }^{19}$ Permanent address: Kangnung National University, Kangnung, Korea.

${ }^{20}$ Now at The Johns Hopkins University, Baltimore, MD 21218, U.S.A.

${ }^{21}$ Permanent address: Sung Kyun Kwan University, Suwon, Korea.

${ }^{22}$ Supported by the US Department of Energy, grant DE-FG03-92ER40689.

${ }^{23}$ Now at Harvard University, Cambridge, MA 02138, U.S.A.

${ }^{24}$ Also at Istituto di Matematica e Fisica, Università di Sassari, Sassari, Italy.

${ }^{25}$ Now at Princeton University, Princeton, NJ 08544, U.S.A.

${ }^{26}$ Now at Max-Planck-Institüte für Kernphysik, Heidelberg, Germany.
} 


\section{Introduction}

There have now been several studies of quark and gluon jet properties published by the LEP Collaborations [1, 2, 3, 4]. In general these have been based on specific configurations of three-jet events, in particular onefold and threefold symmetric events. As a result of constraining the event topology the full two-dimensional ${ }^{1}$ phase space for three-jets is effectively reduced to a one-dimension curve, or even a point. Additionally such restrictions limit the available statistics. In this paper the earlier analyses are extended in a study of the behavior of the mean charged particle multiplicity of quark jets, gluon jets and whole events for all three-jet configurations.

A principle result of this work is a demonstration that the energy of a quark or gluon jet is an inappropriate scale to uniquely specify its mean multiplicity. That is, samples of quark (or gluon) jets of identical energy generally have different mean multiplicities. New energy- and topology-sensitive scales are suggested and shown to better describe the jet data. This result is confirmed in a quantitative analysis of whole-event multiplicities where it is not necessary to apply tagging or assign tracks to individual jets. In previously published LEP analyses [1-4] only dependences on energy were considered.

The idea that the position of a jet relative to the other jets, in addition to its energy, is important in determining its properties follows naturally from the coherence of QCD radiation [5]. This should particularly be true for the event multiplicities which are dominated by long wavelength, soft gluons [6]. Colour charge is conserved by QCD so that at leading order in $\alpha_{s}$ and the number of colours, the large $N_{c}$ limit, the gluon carries an equal and opposite anti-colour charge to that of the quark; likewise for the anti-quark. This is illustrated in figure 1.
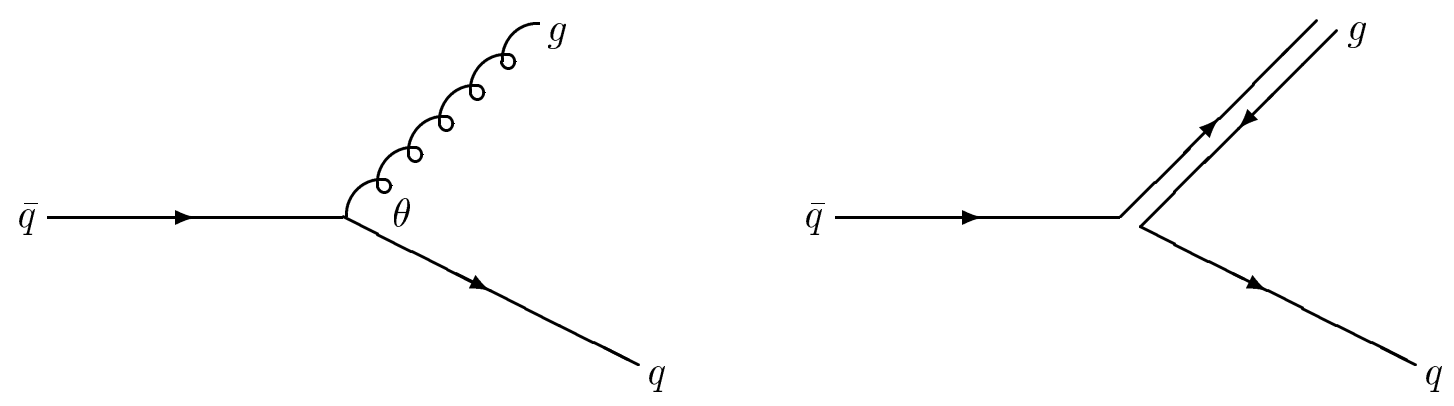

Figure 1: A three-jet event and the colour flow to $\mathcal{O}\left(\alpha_{s} N_{c}^{2}\right)$.

It is therefore natural to expect that as the quark and gluon, for example, come close to each other their colour charges mutually shield one another and the amount of subsequent gluon radiation is reduced. The quark and gluon are not independent but form a coherent system. Monte Carlo event generators such as HERWIG [7] and JETSET [8] which work within the Modified Leading Logarithm Approximation (MLLA) to perturbative QCD (pQCD) build in these coherence effects. Indeed it can be argued that the radiating units are not the actual partons but rather the colour dipoles formed between them [9]; this approach is directly exploited in the ARIADnE [10] Monte Carlo. The analysis presented here confirms that the data do not support the concept of independent jets but prefer a more coherent description.

\footnotetext{
${ }^{1}$ The event plane orientation is not expected to play a rôle in this analysis and is not therefore considered here.
} 
In section two of this paper the experimental procedures used to select and correct the data sets are detailed. In section three new topology-sensitive scales are introduced and used to demonstrate that they can characterize quark and gluon jet mean multiplicities whilst energy alone cannot. In section four these new scales are used to parameterize the jet and whole-event charged multiplicities in three-jet events. Finally conclusions are given in section five.

\section{Detector, data selection}

\subsection{Detector}

The ALEPH detector, which provides both tracking and calorimetric information over almost the full solid angle, is described in detail elsewhere [11]. The momenta of charged particles are obtained from a fit to information provided by the three tracking devices: a two-layer, double-sided silicon vertex detector, an eight-layer, axial-wire drift chamber and a large time projection chamber. This measurement, when combined with calorimeter and muon chamber information allows the reconstruction of energy flow objects [12]. Taking advantage in this way of the redundancies built into the apparatus improves both the energy and the angular resolutions.

\subsection{Selection of three-jet events}

The results presented here are based on two data samples which are used in the wholeevent and single-jet studies. In each case hadronic $Z$ decays are selected using the standard ALEPH criteria [13]. The Durham jet clustering algorithm [14] with a resolution parameter of $y_{\text {cut }}^{D}=0.01$ is then used to further select three-jet events. Geometric cuts are applied to ensure that the event is well contained within the detector. The jet energies are recomputed from their relative opening angles assuming massless, planar kinematics; this significantly improves the resolution obtained on the jet energies.

The whole-event sample is based on $1.8 \times 10^{6}$ hadronic $Z$ decays. The three jet selection is as described above with in addition a minimum $30^{\circ}$ inter-jet angle. This results in $3 \times 10^{5}$ three-jet events, which have no tagging or unfolding applied.

The quark and gluon jet properties are measured in a sample of $5.4 \times 10^{5}$ three-jet events. First, a $b \bar{b} g$ enriched subsample of $5 \times 10^{4}$ events is obtained using an impact parameter tag [15]: the probability that all the charged tracks originate from the primary vertex is required to be less than $10^{-3}$. The gluon jet is identified as the jet with the highest probability to be from the primary vertex. The resulting purity of the gluon jet varies between $50 \%$ at a jet energy of $40 \mathrm{GeV}$ to $97 \%$ at $5 \mathrm{GeV}$ and is typically larger than $90 \%$. The gluon jet charged multiplicities are determined as a function of its energy and also the angles to the other two jets. This binning allows any dependence on event topology to be investigated. The effect of the residual quark contamination is removed by applying a small, $\leq 8 \%$, multiplicative correction factor determined from Monte Carlo. Second, charged particle multiplicities in quark jets are linearly unfolded from the full, untagged, three-jet event sample using the above gluon results and quark/gluon compositions determined from the leading order QCD matrix element. This unfolding is possible because the gluon jets are the same in $u d s c$ - and $b$-quark initiated events, as expected from QCD. 


\subsection{Purities}

After selecting a three-jet event, one is faced with the issue of assigning to each jet probabilities for the quark and gluon hypotheses. At $\mathcal{O}\left(\alpha_{s}^{2}\right)$ and beyond this classification is a matter of definition. Two prescriptions have been used in this paper according to the information available.

Matrix Element method: Using only the jet energies the leading order QCD matrix element may be used to give a probability that any particular jet is the gluon [18]:

$$
\mathcal{P}_{3=g} \propto \frac{x_{1}^{2}+x_{2}^{2}}{\left(1-x_{1}\right)\left(1-x_{2}\right)}
$$

and so on, where $x_{i}$ are the three scaled jet energies: $x_{i}=2 E_{i}^{\mathrm{jet}} / E_{\mathrm{cm}}$. This definition does not require any tagging.

Monte Carlo matching method: An alternative method based on fully simulated Monte Carlo events is to use an angular matching procedure to pair sets of three jets found at detector level to three jets at the end of the parton shower. If the two primary quarks can be unambiguously traced to two separate jets at detector level then the third jet is called a gluon jet. Such events allow probabilities to be defined for jets in specific configurations which can be applied to data. This procedure is particularly important when a flavour tag indicates the presence of a heavy quark in one or more of the jets; variants of this method have been extensively used in previous studies [1-4].

A comparison between the gluon jet purities obtained using the matrix element and the Monte Carlo matching definitions has been performed as a function of the event topology. For a jet of energy $E_{\text {jet }}$ the variable chosen to characterize the event topology is $\Delta \theta=\theta_{\max }-\theta_{\min }$ the difference of the opening angles to the other two jets ${ }^{2}$. The variable $\Delta \theta$, which is independent of $E_{\text {jet }}$, has several simple properties: $\Delta \theta=0$ for symmetric events and $\Delta \theta=180^{\circ}$ for two-jet-like events. The variables $\left(E_{\text {jet }}, \Delta \theta\right)$ span a rectangular phase space $0<E_{\text {jet }}<E_{\mathrm{cm}} / 2$ and $0<\Delta \theta<180^{\circ}$; selecting jets with $y_{\text {cut }}^{D}=0.01$ and a minimum inter-jet angle restricts the analysis to a subspace with curved boundaries.

Figure 2 shows the lines of constant gluon purity in the $\left(E_{\text {jet }}, \Delta \theta\right)$ phase space. The full lines are obtained with the matrix element definition and the dotted lines with the Monte Carlo matching. The agreement between the two definitions is good except at the phase space boundaries. This graph also confirms that the jet energy $E_{\text {jet }}$ essentially fixes the gluon purity and thus that different topologies for jets of fixed energy can be studied at almost constant gluon purity. The scheme based on the leading order QCD calculation has been used in this paper for quark/gluon jet purity evaluation in three-jet events. It has the advantage of avoiding the complexity and the uncertainties involved with the Monte Carlo matching method.

\subsection{Acceptance corrections and checks}

For each sample the effects of geometrical acceptance, detector efficiency and resolution are obtained by comparing the properties of Monte Carlo events before and after detector simulation. From an event generator based on DYMU3 [16] and JETSET 7.3 [8] with parameters adjusted to describe the ALEPH data [13] are obtained $2.8 \times 10^{6}$ hadronic events. These events are passed through the full ALEPH detector simulation. After event reconstruction three-jet events are selected as for the data. This allows multiplicative

\footnotetext{
${ }^{2}$ By kinematics fixing the jet's energy strongly constrains the opening angle between the other two jets.
} 
corrections to be determined which are applied bin-by-bin on any measured raw data distribution, according to the method explained in reference [13]. The definition of charged multiplicity follows [17]: all particles with lifetimes $<1$ ns are forced to decay; this includes $K_{S}^{0}$ 's and strange baryons, whilst tracks associated with photon conversions are not counted. These corrections factors are small, $\leq 8 \%$, and do not alter the basic shapes of the measured raw distributions.

Several checks of the correction/unfolding method used to obtain the the quark and gluon jet properties were carried out. The results were shown to be stable against altering $y_{\text {cut }}^{D}$ to 0.005 or 0.02 , or imposing a minimum, $70^{\circ}$, angular separation between the jets. In the $b \bar{b} g$ enriched sample the gluon jet identification was changed to require it to have a probability that its tracks come from the primary vertex $>0.2$ and the other two jets $<0.1$ before applying a correction for quark contamination. For the quark jet sample the unfolding was repeated using the Monte Carlo matching definition of purity, described above. In both cases the resulting distributions remained very similar. Finally tests were also performed to explicitly demonstrate using the data that the gluon jet properties in the $b$-tagged and untagged event samples are equivalent.

\section{Topology sensitive scales}

\subsection{Quark scale}

The idea that the gluon and (anti-)quark colour charges screen each other suggests that the appropriate scale for quarks (and likewise antiquarks) is

$$
Q_{q g}=E_{q} \sin \left(\frac{\theta_{q g}}{2}\right)
$$

rather than $Q_{E}=E_{q}$. Here $E_{q}$ is the quark's energy and $\theta_{q g}$ the quark-gluon opening angle. This scale depends only on the direction (and not energy) of the gluon and tends to $E_{q}$ when the quark and gluon are back to back, i.e., colour screening is minimal, and vanishes as the quark and gluon become parallel, i.e., maximal colour screening. The scale $Q_{q g}$ is related to the quark's transverse momentum with respect to the gluon direction. In practice one cannot per se say which is the gluon jet but $\theta_{\min }$, the angle to the closest jet, predominantly gives the desired angle: $\theta_{\min } \approx \theta_{q g}$. The probability that this assignment is correct lies in the range $75-95 \%$. In figure 3 the mean quark jet charged multiplicity, $\left\langle N_{q}\right\rangle$, is plotted as a function of the two quark scales: a) $Q_{E}=E_{q}$ (figure 3.a) and b) $Q_{\text {min }}=E_{q} \sin \left(\theta_{\min } / 2\right) \approx Q_{q g}$ (figure 3.b). In figure 3.a the points in a vertical band all lie in the same energy bin but different $\Delta \theta$ sub-bins: they have been plotted such that a lower energy point corresponds to a lower $\Delta \theta$ bin. Each data point in figure 3.a corresponds to a single value of $Q_{\min }$. A clear difference is the extent to which the data points lie on a single curve, i.e., the extent to which the scale uniquely determines the mean charged multiplicity for quark jets. At fixed $Q_{E}$ the vertical dispersion in figure 3.a is a measure of the unaccounted-for topology dependence, whereas figure 3.b shows little dispersion. To confirm the effectiveness of the new scale a single function taken from the (phenomenological) set of functions $\left\{a+b \log Q+c \log ^{2} Q\right\}$ is tested to see how well it is able to describe the data. Performing a fit the best chi-squared per degree of freedom $\left(\chi^{2} /\right.$ d.o.f.) for $Q=Q_{E}$ is $1208 /(59-3)=21.6$ whilst for $Q=Q_{\min } \approx Q_{q g}$ it is $54 /(59-3)=0.96$. The dashed curve in figure 3.b shows this best fit function; the solid curve is described in section 4 . The ad hoc scales $Q_{\max } \approx Q_{q \bar{q}}$ and $\bar{Q}_{g}$ (see equation (4) 
below) also fail to describe the data: their minimum $\chi^{2} /$ d.o.f. are $893 /(59-3)=15.9$ and $235 /(59-3)=4.20$, respectively.

\subsection{Gluon scales}

In the previously advocated picture the gluon is approximated by a pair of colouranticolour charges so that using two scales seems appropriate to describe a gluon, one associated with each colour line. This leads to the following expression for the two scales:

$$
Q_{g q}=E_{g} \sin \left(\frac{\theta_{g q}}{2}\right) \quad \text { and } \quad Q_{g \bar{q}}=E_{g} \sin \left(\frac{\theta_{g \bar{q}}}{2}\right) .
$$

As explained in the next section, these two scales can be combined into one effective scale:

$$
\bar{Q}_{g}=\sqrt{Q_{g q} Q_{g \bar{q}}}=\sqrt{Q_{\max } Q_{\min }} .
$$

The mean gluon jet charged multiplicity, $\left\langle N_{g}\right\rangle$, is plotted in figure 4 as a function of: a) $Q_{E}=E_{g}$ and b) $Q=\bar{Q}_{g}$. Again significant vertical dispersion is apparent in the data points belonging to the same energy $\left(Q_{E}\right)$ whereas the data align on a single curve for the topological scale $\left(\bar{Q}_{g}\right)$. Performing a least-squares fit, assuming a quadratic dependence on $\log Q$, yields a $\chi^{2} /$ d.o.f. for $Q=Q_{E}$ of $373 /(59-3)=6.66$ whilst for $Q=\bar{Q}_{g}$ it drops to $39.0 /(59-3)=0.70$. The ad hoc scales $Q_{\min }$ and $Q_{\max }$ also fail to parameterize the data: their minimum $\chi^{2} /$ d.o.f. are $263 /(59-3)=4.70$ and $388 /(59-3)=6.93$, respectively.

This result is confirmed in an independent study performed using a highly-enriched sample of gluon jets which avoids using model-based purity corrections and instead relies on stricter selection criteria. The event selection is similar to that of the nominal analysis but now includes tighter geometrical cuts to insure that all three jets are contained well within the coverage of the vertex detector. The tagging procedure is again based on the impact parameter technique [15]. Two of the three jets are tagged as heavy flavour jets, the third is taken as the gluon jet. The tagging criteria are energy dependent in order to optimize the selection efficiency whilst maintaining high gluon purities across the whole available jet energy range. This yields $1.1 \times 10^{3}$ jets whose gluon purity lies between 73 and $97 \%$ according to their energy, with a $94 \%$ average value. No correction was applied for the small residual quark contamination or even detector acceptance. The same investigation of the scale dependence of the mean charged multiplicity is repeated. Assuming a quadratic dependence on $\log Q$ the $\chi^{2} /$ d.o.f. $=79 /(49-3)=1.72$ for the scale $\bar{Q}_{g}$ and $281 /(49-3)=6.11$ for the scale $Q_{E}$; again the mean charged multiplicity is found to be a function of $\bar{Q}_{g}$ and not $Q_{E}$.

\section{Jet and Event Multiplicities}

In the previous section topology-sensitive scales are identified, $Q_{\min }$ and $\bar{Q}_{g}$, which are more appropriate than the jet energy for characterizing mean charged multiplicities in jets. These scales are now used to parameterize the variations of jet and whole event multiplicities.

\subsection{Multiplicity formulae}

The evolution of the mean charged particle multiplicity in $e^{+} e^{-}$collisions, as a function of center of mass energy, has been reliably predicted in pQCD by applying Local Parton- 
Hadron Duality to a MLLA calculation [6]:

$$
\left\langle N^{e^{+} e^{-}}\right\rangle(Q)=2 K \alpha_{s}^{b}(Q) \exp \left(\frac{c}{\sqrt{\alpha_{s}(Q)}}\right) \cdot\left(1+\mathcal{O}\left(\sqrt{\alpha_{s}}\right)\right) \equiv 2 \mathcal{N}(Q)
$$

with

$$
\alpha_{s}(Q, \Lambda)=\frac{4 \pi}{\beta_{0} \log \left(Q^{2} / \Lambda^{2}\right)}\left[1-\frac{\beta_{1}}{\beta_{0}^{2}} \frac{\log \log \left(Q^{2} / \Lambda^{2}\right)}{\log \left(Q^{2} / \Lambda^{2}\right)}\right] .
$$

For five active flavours [6], $b \approx 0.492, c \approx 2.265, \beta_{0} \approx 7.667$ and $\beta_{1} \approx 38.67, Q$ is the 'energy' scale, $K$ and $\Lambda$ are free parameters ${ }^{3}$ which must be found from fits to event charged multiplicity data. Using half the charged multiplicity in $e^{+} e^{-}$annihilation events, compiled in [22], and the scale $Q=\sqrt{s} / 2$, which is in agreement with equation (2), reference values of $K$ and $\Lambda$ are determined. The result of the fit is $K=0.035 \pm 0.004$ and $\Lambda=80 \pm 29 \mathrm{MeV}$ where $K$ and $\Lambda$ are almost fully correlated. Hadronic $Z$ decays are dominated by pairs of back-to-back quark jets so that to leading order $\left\langle N^{e^{+} e^{-}}\right\rangle=2\left\langle N_{q}\right\rangle$; thus equation (5) is used to also describe the mean charged multiplicity of quark jets: $\left\langle N_{q}\right\rangle(Q)=\mathcal{N}(Q)$

The mean multiplicity in a pair of back-to-back gluon jets has also been calculated and at leading order (LO) is predicted to be approximately twice $\left(C_{A} / C_{F}\right)$ that of an equivalent pair of quark jets [19]. However large corrections to this ratio are expected at Next-toNext-to-Leading Order [20] and particularly when energy conservation is implemented [21], so that $\left\langle N_{g}(Q)\right\rangle=f \mathcal{N}(Q)$ is used with $f$ free. In realistic situations it has been argued that it is more appropriate to consider the gluon as having two scales, leading to the following expression for the mean charged multiplicity: $\left\langle N_{g}\right\rangle=f\left[\mathcal{N}\left(Q_{g q}\right)+\mathcal{N}\left(Q_{g \bar{q}}\right)\right] / 2$. Now since the scale dependence of the jet multiplicity is expected to be via $\alpha_{s}(Q)$ and hence logarithmic the two scales for a gluon can be combined into one, equation (4), and the multiplicity given by: $f \mathcal{N}\left(\bar{Q}_{g}\right)$. To see this consider $\left\langle N_{g}\right\rangle=\mathcal{N}\left(Q_{g q}\right)+\mathcal{N}\left(Q_{g \bar{q}}\right)$ and write $Q_{g q}=\bar{Q}_{g} \times \sqrt{Q_{g q} / Q_{g \bar{q}}}$ so that $\log Q_{g q}=\log \bar{Q}_{g}+\log \sqrt{Q_{g q} / Q_{g \bar{q}}}$, likewise for $Q_{g \bar{q}}$; then expanding each $\mathcal{N}$ in a Taylor series one has $\left\langle N_{g}\right\rangle=2 \mathcal{N}\left(\bar{Q}_{g}\right)+\mathcal{O}\left(\mathcal{N}^{\prime \prime}\right)$. This result only assumes a logarithmic scale dependence in $\mathcal{N}(Q)$. When the previously employed

quadratic form is used the difference between the two expressions is $2 c \log ^{2} \sqrt{Q_{g q} / Q_{g \bar{q}}}$. If equation (5) is used for $\mathcal{N}$ then the neglected term is of higher order than the presently calculated terms [6] and it would be unjustified to retain it.

It is important to remember that using the above functional form for the multiplicity in an individual jet is an approximation to the full theory since it takes no account of how the jet definition chooses to assign particles to the jets. However it should offer a reasonable first estimate for the purpose of comparison. Alternatively one can regard equation (5) as simply a parameterization of the, two-jet dominated, total event multiplicity to which comparison is made.

\section{$4.2 \quad$ Jet multiplicities}

Equation (5) is used to try and fit the mean quark and gluon jet charged multiplicities of the unfolded/corrected data samples, determined in subsections 3.1 and 3.2, with the energy scale definitions given in equations (2) and (4), respectively. The equation fails to describe the quark jet data, as can be seen in figure 3 where the solid curve represents

\footnotetext{
${ }^{3}$ The full $\sqrt{\alpha_{s}}$ correction to equation (5) would need to be known before the effective $\Lambda$ appearing in the multiplicity formula could be related to that determined from other measurements.
} 
the best fit. However the choice of scale is the correct one and it is the choice of the functional form for the scale dependence which is wrong. As previously observed a good fit is possible by assuming a quadratic dependence on $\log Q$, the dashed line in figure 3 . In contrast the gluon jet data is described very well by equation (5) with fitted parameters $f K=0.046 \pm 0.004$ and $\Lambda=80 \pm 16 \mathrm{MeV}$; this is the solid line in figure 4 .

\subsection{Event multiplicities in three-jet events}

Rather than assigning particles to jets according to a cluster jet algorithm and study the exclusive jet mean charged multiplicities, the fully inclusive total multiplicity is now considered. This simpler observable has the advantage of minimizing any sensitivity to the choice of jet algorithm [2,4] and is likely to be more amenable to a theoretical description. Further, in order to avoid biases in the tagged jet properties, only the events of the untagged event sample described in subsection 2.2 are used; no $b$ tagging and thus no unfolding is performed. This not only reduces strongly the size of the systematic errors but also gives a substantial gain in statistics.

The total event multiplicity is regarded as consisting of three components $\left\langle N_{\text {evt }}\right\rangle=$ $\left\langle N_{q}+N_{\bar{q}}+N_{g}\right\rangle$. Two expressions for $\left\langle N_{\text {evt }}\right\rangle$ are considered, based on using equation (5) with either jet energies or the topology-sensitive scales introduced in equations (2) and (3). Since in practice it is not known which jet is the gluon all expressions are averaged over the three ways of assigning the gluon weighted by their probabilities. Probabilities based on equation (1) are used for this purpose. The expression using jet energy is:

$$
\left\langle N_{\text {evt }}\right\rangle=\sum_{\text {perms }} \mathcal{P}_{1=g}\left\{f \mathcal{N}\left(E_{1}\right)+\mathcal{N}\left(E_{2}\right)+\mathcal{N}\left(E_{3}\right)\right\}
$$

and the expression using topology-sensitive scales is:

$$
\left\langle N_{\text {evt }}\right\rangle=\sum_{\text {perms }} \mathcal{P}_{1=g}\left\{f \mathcal{N}\left(\bar{Q}_{1}\right)+\mathcal{N}\left(Q_{21}\right)+\mathcal{N}\left(Q_{31}\right)\right\} .
$$

Here $\bar{Q}_{1}=\sqrt{Q_{12} Q_{13}}$ is the gluon scale and $Q_{21}\left(Q_{31}\right)$ is the corresponding quark (antiquark) scale. Figure 5 shows the mean total charged multiplicity of each threejet event in $4 \mathrm{GeV}$ bins of $E_{\text {jet }}$, as a function of $\Delta \theta$, using $20^{\circ}$ bins. Here each event contributes to three bins in the $\left(E_{\text {jet }}, \Delta \theta\right)$ space shown in figure 2 , once as each of the three jets are selected; the sub-figures correspond to vertical slices across this space. Fitting this data allows $K, \Lambda$ and $f$ to be fitted simultaneously. The dashed curves are the results of a fit using the independent jets model, equation (7), where the scale is the jet energy for both quark and gluon jets. Clearly, this choice of scale is excluded, the best fit giving $\chi^{2} /$ d.o.f. $=559 /(65-3)=9.02$. The solid curves show the best fit based on the coherent jets model, equation (8), which uses the new topology-sensitive scales. The fitted parameters in this case are $K=0.034 \pm 0.002, \Lambda=81 \pm 10 \mathrm{MeV}$ and $f=1.48 \pm 0.08$ giving a $\chi^{2} /$ d.o.f. $=56.0 /(65-3)=0.90$. Again $K$ and $\Lambda$ are almost fully correlated to each other whilst $f$ is almost fully anti-correlated to them. If the Monte Carlo matching based definition of purity is instead used in equation (5) then the fit gives almost the same results.

A test of the Monte Carlo models was also performed on the total event multiplicity. ARIADNE, HERWIG and JETSET are all capable of reproducing the three-jet data as a function of position in phase space. Further their predictions may be successfully parameterized using equation (8) with the same values of $K, \Lambda$ and $f$ as found for data. 
In fit results for both the single jet and whole event samples quoted above only the numerically dominant statistical errors are used in the $\chi^{2}$ measure. Many sources of potential systematic error were investigated, including all those considered in section 2.4. Additionally to check stability the fit region used in the complete three-jet study was reduced to only those $4 \mathrm{GeV} \times 20^{\circ}$ bins wholly contained within the three-jet event definition region. Finally instead of numerically integrating the theoretical functions over the allowed regions of the wide bins to obtain predicted average values, the fit was repeated using the function values at the centers of small, $1 \mathrm{GeV} \times 2^{\circ}$, bins. In all cases the measured effects on the fit results were below the level of those due to the statistical errors and in no instance greater than $10 \%$.

\subsection{Discussion}

The QCD derived formula, equation (5), for the scale dependence of mean charged particle multiplicities provides an excellent description of the data for (half) the total multiplicity of all $e^{+} e^{-}$annihilation events, as it should, for the gluon jet data and for the total multiplicity of the untagged three-jet event sample, provided the proposed topological scales are used, equation (8). The best fit values of the free parameters, $K, \Lambda$ and $f$, obtained for these three data sets are remarkably similar, which could be interpreted as evidence for an universality in the hadronization process. It is interesting that the value of the gluon-jet/quark-jet multiplicity ratio obtained, $f \approx 1.5$, is in good agreement with the most recent OPAL measurement [4], which aims at a jet definition independent result, and also the theoretical prediction in reference [21].

However, equation (5) fails badly to describe the observed scale dependence of the quark jet multiplicity. Two features are worthy of comment. The relatively high multiplicities at low scale, compared to half that measured in an $e^{+} e^{-}$annihilation event at a corresponding lower $\sqrt{s}$, and the flattening of the dependence at higher scales, see figure 3.b. Neither feature can be accommodated simultaneously in the QCD based formula, equation (5). Nevertheless it is an important fact that the coherent Monte Carlos, in particular JETSET which was used in the data correction/unfolding, reproduces the behavior of the quark jet multiplicity in detail. That is, the MLLA contains, in large part, the physics underlying this observed scale dependence, unlike equation (5). The high values at low scales cannot be attributed to using incorrect purities when performing the unfolding procedure since in this region, 5-10 GeV, both quarks and gluons have comparable multiplicities; changing the purities would not alter this result.

A conclusion to be drawn from these measurements is that the quark jet multiplicity defined as half that seen in a full $e^{+} e^{-}$annihilation event is not equivalent to the number of tracks assigned to a quark jet by a cluster algorithm. As emphasized previously, a potential reason why equation (5), which was developed to describe the total event multiplicity, fails to describe the quark jet data is that the formula does not contain any information about the jet algorithm. It is then all the more remarkable that the formula works so well for gluon jets. Such sensitivity to the details of the jet definition have been reported previously $[2,4]$.

\section{Conclusions}

Quark and gluon jet mean multiplicities are not functions of jet energy alone, as demonstrated in figures 3.a and 4.a by the large vertical dispersions, up to $40 \%$ at low 
scales. This is due to the neglect of topology dependence. Inspired by the colour coherence

of QCD new transverse momentum-like scales are proposed, $Q_{\min }$ for quarks and $\bar{Q}_{g}$ for gluons, which can uniquely parameterize the data shown in figures 3.b and 4.b.

An investigation of the functional form of the jets' multiplicity dependence on these new scales shows that equation (5), developed to describe total event multiplicities in $e^{+} e^{-}$annihilation, can also describe gluon jets using very similar parameters. This is not the case for quark jets. The form of the scale dependence in a quark jet's multiplicity defined to be half that of an $e^{+} e^{-}$annihilation event or defined with the aid of a cluster jet algorithm in a three-jet event are quite different. These measurements highlight the need for any theoretical prediction to take account of the precise definition of jet multiplicity.

The naïve model for the total event multiplicity, taking full account of the three-jets' relative orientations, is tested. In the coherent jets version, equation (8), where the new scales are used, the model is fully capable of describing the data with a consistent value of $f \approx 1.5$. In contrast the independent jets model, equation $(7)$, is wholly incapable of describing the data, thus providing further confirmation of the need for topology dependent scales, rather than jet energies, when studying quark and gluon jet properties.

\section{Acknowledgements}

We wish to thank our colleagues from the accelerator divisions for the successful operation of the LEP machine and the engineers and technical staff in all our institutions for their contribution to the good performance of ALEPH. Those of us from non-member states thank CERN for its hospitality. 


\section{References}

[1] ALEPH Collaboration: D. Buskulic, et al., Phys. Lett. B346 (1995) 389; and Phys. Lett. B384 (1996) 353.

[2] DELPHI Collaboration: P. Abreu, et al., Zeit. Phys. C70 (1996) 179.

[3] OPAL Collaboration: G. Alexander, et al., Phys. Lett. B265 (1991) 462; P.D. Acton, et al., Zeit. Phys. C58 (1993) 387; and G. Alexander, et al., Zeit. Phys. C69 (1996) 543.

[4] OPAL Collaboration: R. Akers, et al., Zeit. Phys. C68 (1995) 179 and G. Alexander et al., Test of QCD analytic predictions for the multiplicity ratio between gluon and quark jets preprint CERN-PPE/96-116 (to appear in Phys. Lett. B);

see also: J.W. Gary, Phys. Rev. D49 (1994) 4503.

[5] Yu. L. Dokshitzer, V.A. Khoze, A.H. Mueller and S.I. Troyan, Basics of Perturbative $Q C D$ (Editions Frontieres, Gif-sur-Yvette, 1991).

[6] A.H. Mueller, Nucl. Phys. B213 (1983) 85 and Erratum B241 (1984) 141;

B.R. Webber, Phys. Lett. B143 (1984) 501.

[7] G. Marchesini, B.R. Webber, G. Abbiendi, I.G. Knowles, M.H. Seymour and L. Stanco, Comp. Phys. Comm. 67 (1992) 465.

[8] T. Sjöstrand, Comp. Phys. Comm. 82 (1994) 74 and Lund preprint LU-TP 95-20.

[9] G. Gustafson, Phys. Lett. B175 (1986) 453;

G. Gustafson and U. Petterson, Nucl. Phys. B306 (1988) 746.

[10] L. Lönnblad, Comp. Phys. Comm. 71 (1992) 15.

[11] ALEPH Collaboration: D. Decamp et al., Nucl. Inst. Meth. 294 (1990) 121.

[12] ALEPH Collaboration: D. Buskulic et al., Nucl. Inst. Meth. 360 (1995) 481.

[13] ALEPH Collaboration: D. Buskulic et al., Zeit. Phys. C55 (1992) 209.

[14] W.J. Stirling, J. Phys. G17 (1991) 1567;

S. Bethke, Z. Kunszt, D.E. Soper, W.J. Stirling, Nucl. Phys. B370 (1990) 310.

[15] ALEPH Collaboration: D. Buskulic et al., Phys. Lett. B313 (1993) 535.

[16] J.E. Campagne and R. Zitoun, Zeit. Phys. C43 (1989) 469.

[17] ALEPH Collaboration: D. Decamp et al., Phys. Lett. B273 (1991) 181.

[18] Z. Fodor, Phys. Lett. B263 (1991) 305.

[19] K. Konishi, A. Ukawa and G. Veneziano, Phys. Lett. B78 (1978) 243;

see also: S.J. Brodsky and J. Gunion, Phys. Rev. Lett. 41 (1976) 402.

[20] J.B. Gaffney and A.H. Mueller, Nucl. Phys. B250 (1985) 109;

E.D. Malaza and B.R. Webber, Nucl. Phys. B267 (1986) 702. 
[21] I.M. Dremin and R.C. Hwa, Phys. Lett. B324 (1994) 477;

I.M. Dremin and V.A. Nechitailo, Mod. Phys. Lett. A9 (1994) 1471.

[22] M. Schmelling, Physica Scripta 51 (1995) 683. 


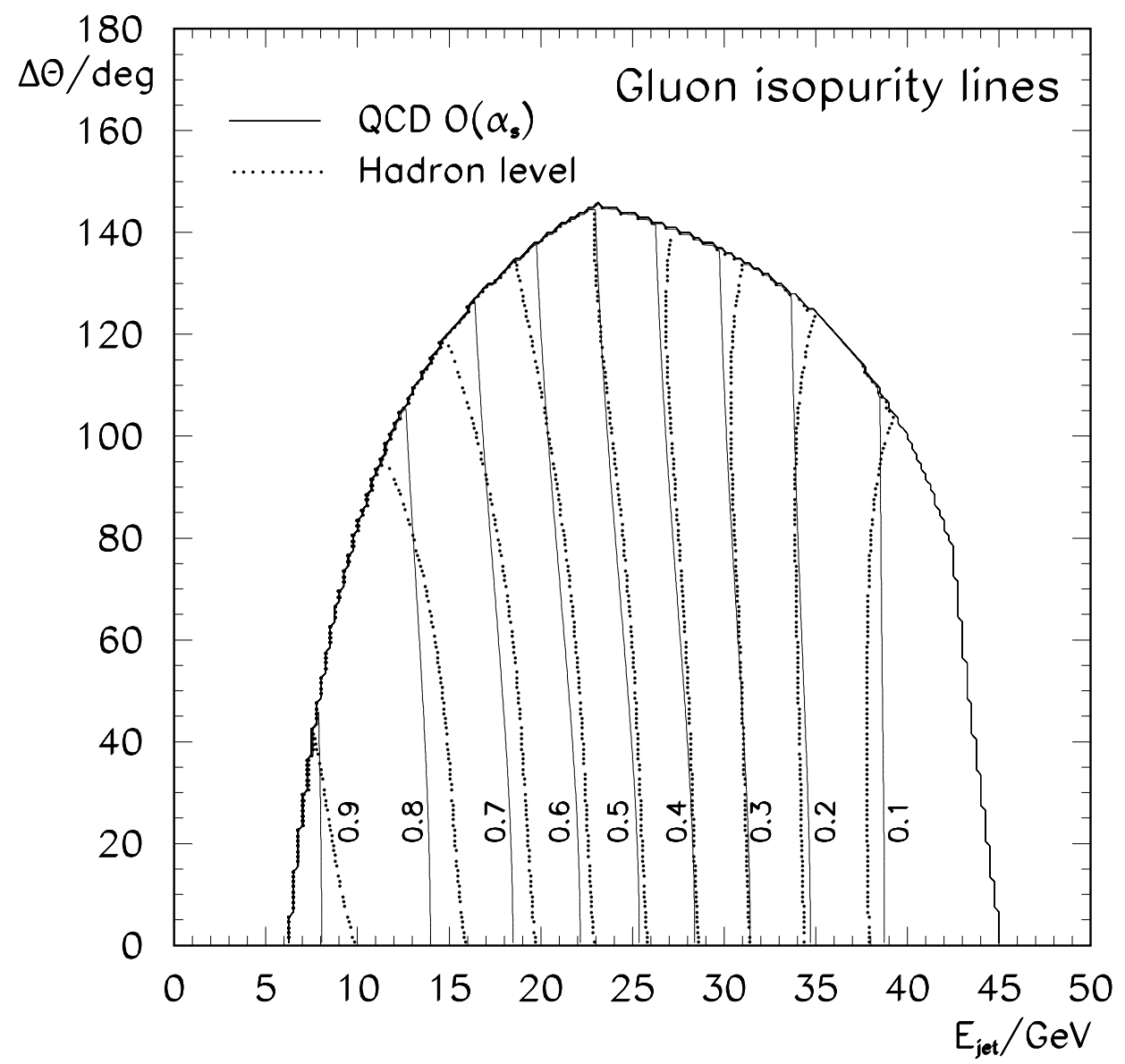

Figure 2: The accessible region of three-body phase space in $\left(E_{\text {jet }}, \Delta \theta\right)$ coordinates after imposing $y_{\text {cut }}^{D}=0.01$. The solid curves show the gluon isopurity lines using the QCD $\mathcal{O}\left(\alpha_{s}\right)$ matrix element based definition and the dotted curves the Monte Carlo matching method. 


\section{Quark Jets}
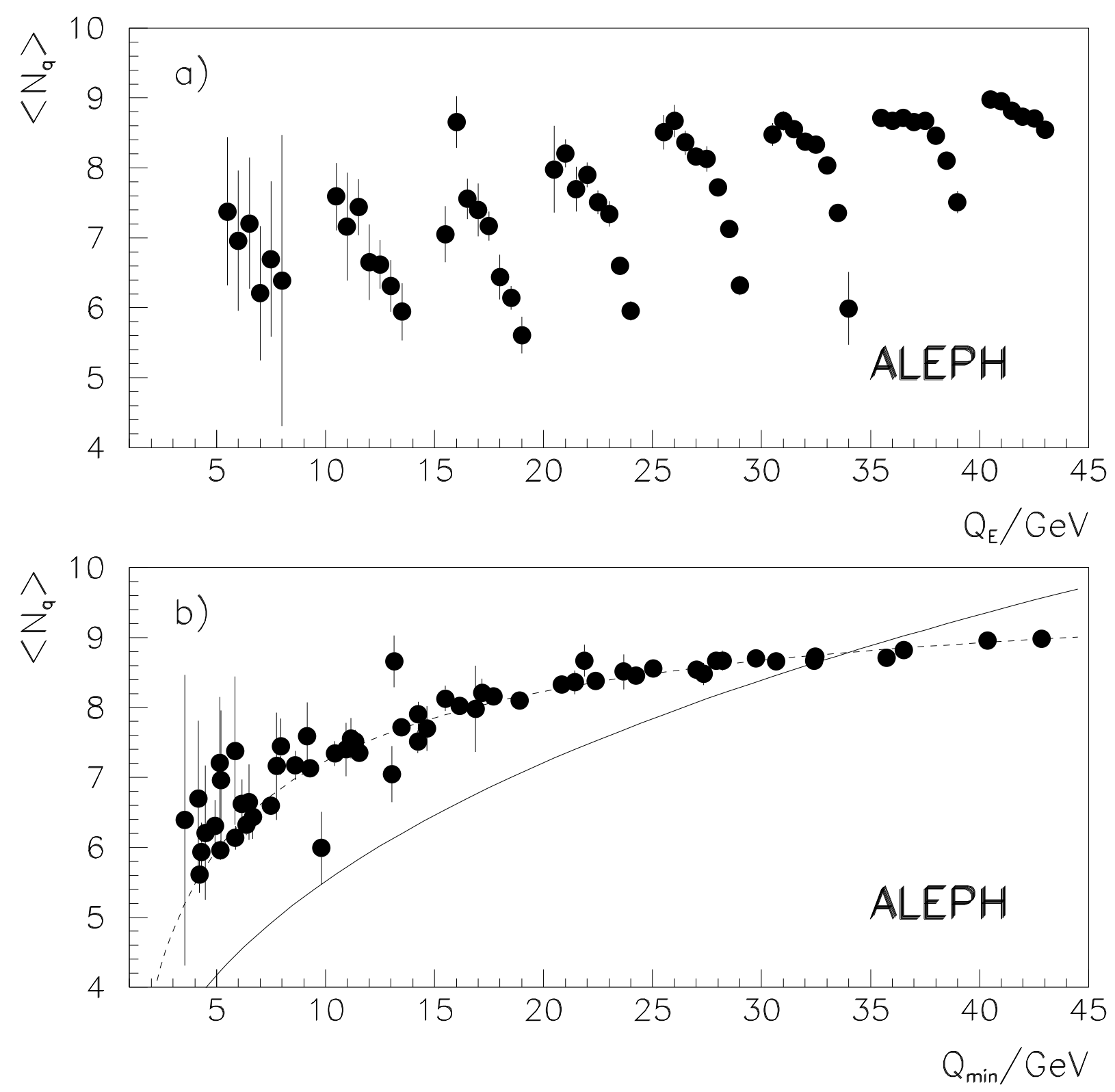

Figure 3: The mean quark jet charged multiplicity plotted as a function of: a) the jet energy, $Q_{E}=E_{q}$, and $\Delta \theta$ or b) the topological scale $Q_{\min }=E_{q} \sin \left(\theta_{\min } / 2\right) \approx Q_{q g}$. In each energy bin the jets are further divided into $\Delta \theta$ sub-bins; in a) lower $\Delta \theta$ bins are shown offset to lower energies and appear as vertical bands. In b) the dashed curve is the best fit to the data assuming a quadratic dependence on $\log Q$, the solid curve is the best fit assuming the QCD-inspired form given in equation (5). The errors shown are statistical only. 


\section{Gluon Jets}
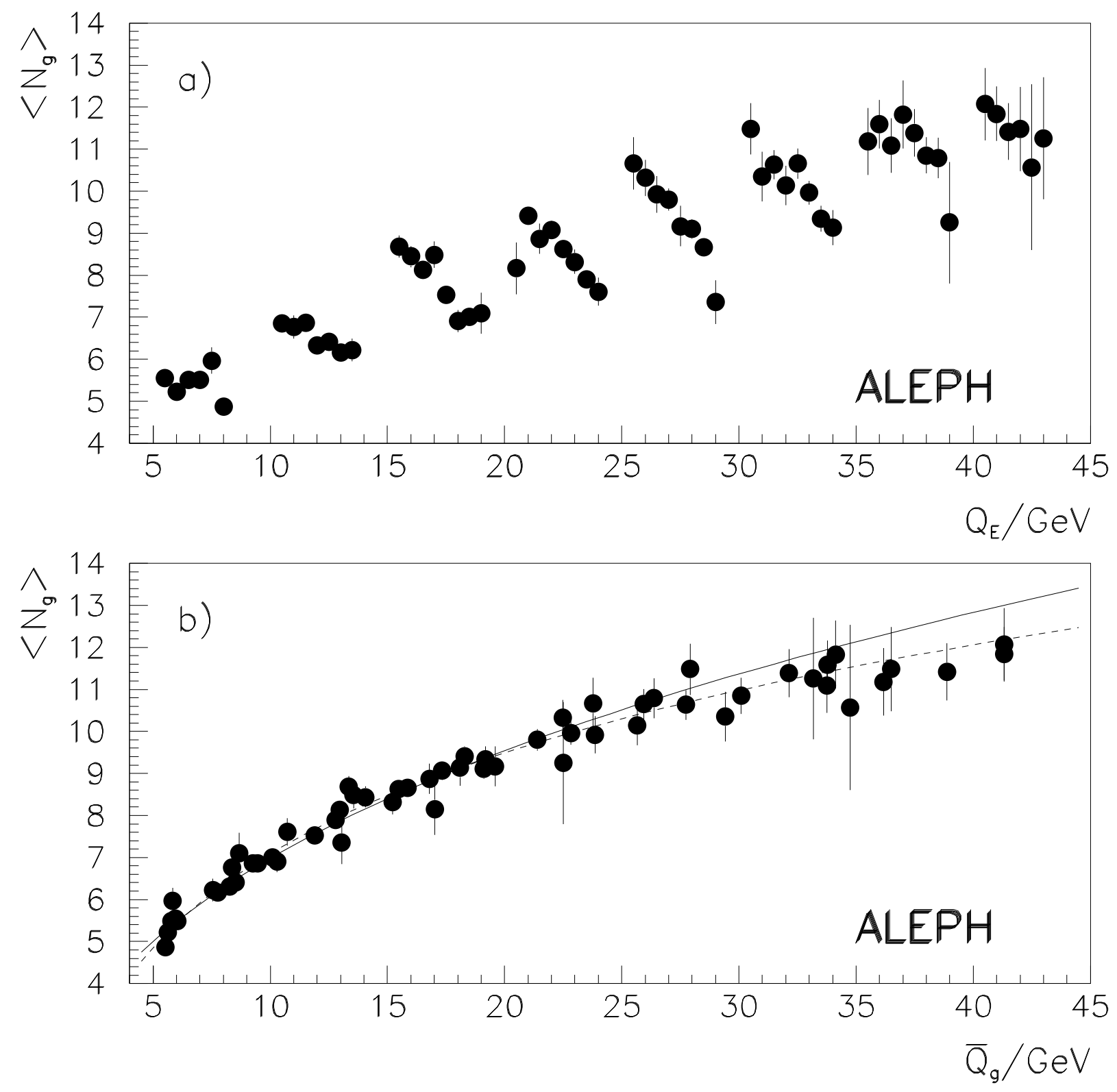

Figure 4: The mean gluon jet charged multiplicity plotted as a function of: a) the jet energy $Q_{E}=E_{g}$ and $\Delta \theta$ and b) the topological scale $\bar{Q}_{g}=\sqrt{Q_{\max } Q_{\min }}$. In each energy bin the jets are further divided into $\Delta \theta$ sub-bins; in a) lower $\Delta \theta$ bins are shown offset to lower energies and appear as vertical bands. In $b$ ) the dashed curve is the best fit to the data assuming a quadratic dependence on $\log Q$, the solid curve is the best fit assuming the QCD-inspired form given in equation (5). The errors shown are statistical only. 


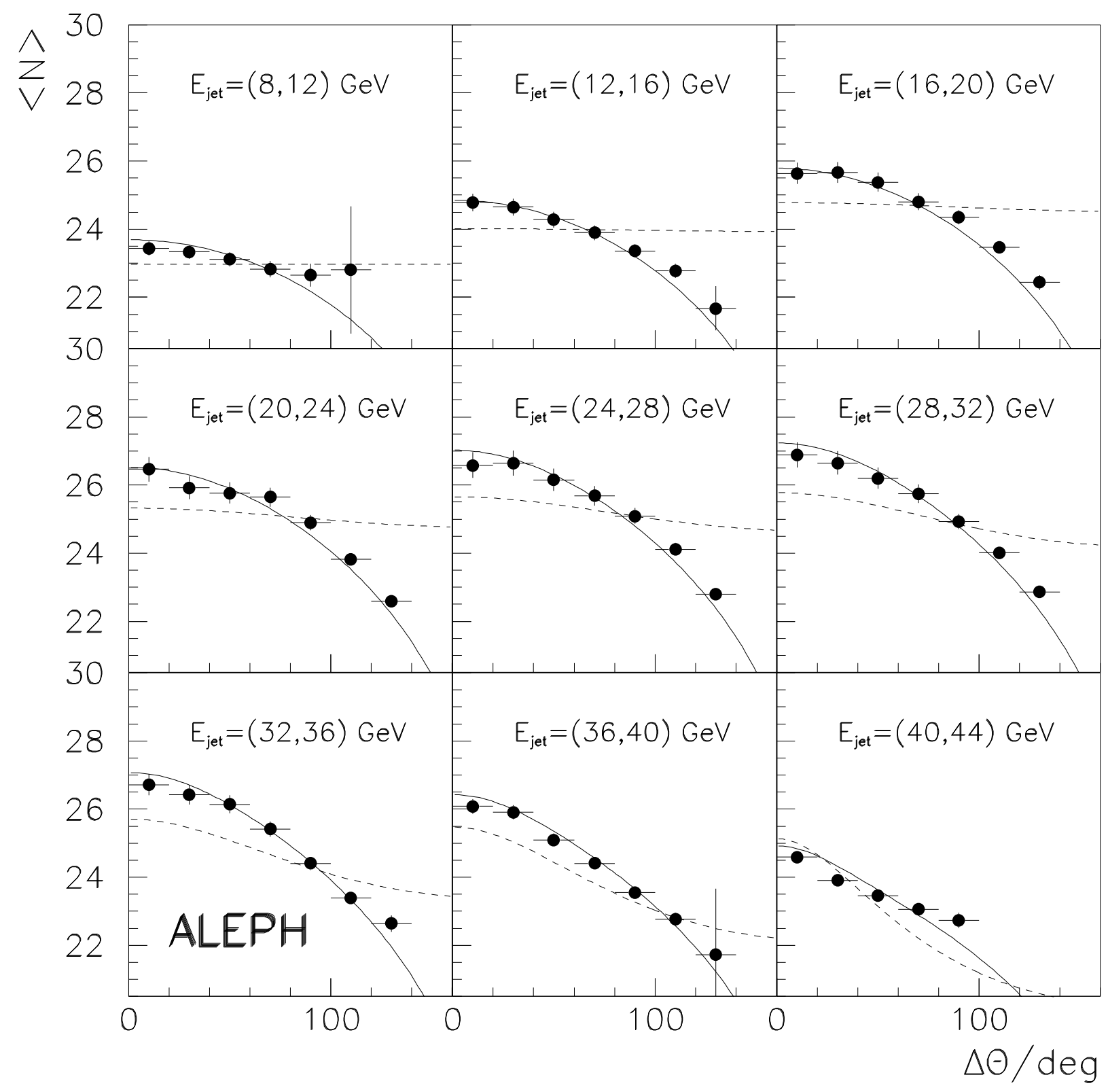

Figure 5: The mean total three-jet event multiplicity plotted as a function of $\Delta \theta=$ $\theta_{\max }-\theta_{\min }$ in $4 \mathrm{GeV}$ bins of the selected jet's energy. The solid curve is the best fit obtained using the coherent jets formula, equation (8), and the dashed curve the best fit obtained using the independent jets formula, equation (7). The errors shown are statistical only. 